\title{
Erratum
}

\section{Correction to van der Ploeg et al., 2017}

The article entitled "From Individual Output to Pooled Data: A Post-Processing Macro for Kubios HRV 2.2" by Melanie M. van der Ploeg, Julian Koenig, Mika P. Tarvainen, and Julian F. Thayer (2017, Journal of Psychophysiology, advance online publication, doi: 10.1027/0269-8803/ a000196) contained an error on p. 3.

The Acknowledgments should read correctly:

\section{Acknowledgments}

The Excel macro on which the updated version is based on, was originally developed by Toby Reel and LaBarron K. Hill in 2007 at The Ohio State University, as indicated in the source code. The Java-based applet [9] was developed by
Andreas Johnsen Lind (Bergen University, Bergen, Norway) and LaBarron K. Hill in 2010.

The authors have no conflict of interest.

The authors regret any inconvenience or confusion this error may have caused.

\section{Reference}

van der Ploeg, M. M., Koenig, J., Tarvainen, M. P., \& Thayer, J. F. (2017). From individual output to pooled data: A postprocessing macro for Kubios HRV 2.2. Journal of Psychophysiology, 1-3. Advance online publication. doi: 10.1027/0269-8803/ a000196 